\title{
The effect of lying upon foot and leg movement
}

\author{
SANDRA M. SCHNEIDER and B. L. KINTZ \\ Western Washington University, Bellingham, Washington 98225
}

\begin{abstract}
A video camera was used to record foot and leg movements of 14 male and 16 female students while (1) engaged in normal conversation with a confederate and (2) while lying to the confederate. Changes in the amount of foot and leg movement when changing from Situation 1 to Situation 2 constituted the experimental data. Both female and male students showed marked changes in foot and leg movement when lying. The changes were of two kinds: more movement while lying or less movement while lying. There was no obvious explanation for which people would move either more or less or why a person would adopt one mode of change rather than the other.
\end{abstract}

Hartshorne and May (1928) concluded that lying by children is more a function of the situation than of the individual. Most children will lie under certain conditions, few will lie under certain other conditions. Kintz (1975), using a questionnaire similar to that of Hartshorne and May, found that college students who scored high in "lying" responded more quickly and made more incorrect responses on a verbal learning task than those with low "lying" scores. These results suggest that, under some conditions at least, there is a detectable characteristic of lying. Christie and Geis (1970) have developed a trait theory about a personality characteristic which they call "Machiavellianism." High-Mach persons are characterized as being manipulators, leaders, and individualists. They are reluctant to cheat at the behest of others, but do so whenever they themselves deem it to be desirable. Exline, Thibaut, Hickey, and Gumpert (1970) found that, when caught cheating, high-Mach persons more vigorously denied any wrongdoing than did the low-Mach individuals.

The data have not been unanimous in showing a tendency toward lying as being a quantifiable trait. Matarazzo, Wien, Jackson, and Manaugh (1970) measured the amount of speech uttered by persons when lying or not. They found no difference in the mean amounts of speech. Matarazzo et al. also found no differences between liars and truth tellers in the amount of eye contact. However, Burns and Kintz (1976) found an interaction in the eye-contact measure between the sex of the subjects and lying. Males increased their eye contact while lying, but the females' eye contact decreased. The overall eye-contact time was about the same for the lying vs. truth-telling conditions, but the pattern for males differed from that of females.

Ekman and Friesen (1969) suggested that a fertile field for lying research would be in the ways in which foot and leg movements betray lying. Most people learn to control speech patterns and facial movements in social situations. Even the hands are carefully monitored to that no obvious movements will correlate with a lie. But the feet and legs are pretty much out of sight and out of consciousness, and therefore may more accurately portray the emotions that are involved with lying.

The present experiment was designed to investigate whether foot and leg movements would change as a function of the two variables: sex and lying.

\section{METHOD}

\section{Subjects}

The subjects were 14 male and 16 female students enrolled in introductory psychology courses at Western Washington University.

\section{Apparatus}

A televesion camera was placed in a room adjacent to the experimental room. The interactions between the student and a confederate were videotaped through a one-way mirror.

\section{Procedure}

The student subject and the experimenter's assistant arrived at the experimental room together. The student was directed to sit in a chair visible through the one-way mirror; the confederate was instructed to sit in a chair facing the student. After a few minutes of conversation intended to put the student at ease, both were informed of the research task. They were asked to try to remember the plots, main characters, and the actors of some recent movies they had seen. After making sure that they understood the request and felt ready, they were instructed to begin.

The period of interaction was taped to establish a baseline rate of foot and leg movement with which to compare a later 3min period when the student would be lying to the confederate. The assistant attempted to structure the conversation so that the student was speaking at least $50 \%$ of the time. There was a series of predetermined questions to help the confederate stimulate the conversation in case the student was not very talkative. After $3 \mathrm{~min}$ of taping had been completed, the student was allowed to overhear a conversation between the experimenter and confederate, to the effect that another reel of tape would be needed. The confederate was sent on the errand to get the tape. The student was told that the real purpose of the discussion was to get rid of the other "student" so that the experimenter could complete the preparations. "This time I want you to lie to the other person so that I can observe bodily reactions to your lies. I want to determine if I can tell if the person is at all aware of the fact that you are not telling the truth. I want you to keep discussing movies and to insert lies into your discussion. Try to 
make sure that your lies will not be detected, that is, don't be obvious about it. Express opinions that you do not really feel, but be careful that the opinions are not too different from those already expressed. Or you can make up the plot of a movie that you have seen."

After insuring that the students understood the request, they were helped to decide in what way they would lie. After giving the students a few minutes to decide whether or not to comply with the request, they were encouraged to practice the lies before the assistant arrived. After giving the students another few minutes of preparation, and after all of their questions had been answered, the confederate returned to the experimental room.

No student refused to lie during the second interval, although some individuals asked several questions. While the students were lying, the confederate acted doubtful about the validity of some of the students' statements by asking probing questions, raising eyebrows, and laughing at the more ridiculous lies. The purpose was to keep the students aware of the fact that they were lying and to try to get them to work hard at being believed.

After the second interview, both of the "subjects" were debriefed. Because it was important that the real reason for the experiment be kept secret from future students, it was explained that no more information about the experiment could be furnished at that time. They were asked not to discuss the experiment with any other students so that future subjects would enter the experiment with no knowledge of it beforehand. They they were given a telephone number and address so they could call at the end of the quarter to find out the details of the experiment that were not given at that time. In addition, it was requested that they furnish their telephone numbers and addresses so that total debriefing could be assured. After thanking both of them for their participation, the student and confederate left together.

\section{RESULTS}

The videotapes were replayed and observed in order to count the students' foot and leg movements. Every time the student made a movement, it was tallied on a piece of paper. Data were gathered for both 3-min intervals. A unit of movement was defined as any movement of the leg or foot as described in an unpublished study (McIntyre \& Kintz, Note 1) in which leg and foot movements were found to be an indication of a topic's saliency for females. The unit of movement ended when it changed direction or stopped. Movements that were continuous and that did not clearly change direction, such as moving the foot in a figure-eight pattern, ended when a circle was completed, the movement stopped, or there was a clear change in the direction of the movement. Simultaneous movements of both limbs counted as one unit.

Since the tapes could be stopped, rewound, and examined with close scrutiny, the counting of movements was done with great accuracy. Each of the two experimenters made a separate count of the units. The interrater reliability, which was estimated by computing a correlation between the two raters, was .99 .

The data were analyzed using a two-variable mixed ANOVA (Type 1). There was no interaction and no difference between the sexes or between the conditions of lying and telling the truth (alpha $=.01$ for all hypotheses). The mean number of movements, for lying vs.
Table 1

Mean Foot and Leg Movements During the $3 \mathrm{~min}$ of Recorded Activity

\begin{tabular}{lcc}
\hline \multirow{2}{*}{$\begin{array}{c}\text { Experimental } \\
\text { Condition }\end{array}$} & \multicolumn{2}{c}{ Sex of the Students } \\
\cline { 2 - 3 } & Male (14) & Female (16) \\
\hline Conversation & 54.79 & 23.16 \\
Lying & 49.39 & 34.75 \\
\hline
\end{tabular}

normal conversation, for the two sexes are presented in Table 1. The apparent differences were well within the range of chance variation.

These comparisons were intended to show whether males or females moved their legs and feet at different rates and whether the mean movement rates were different when lying or conversing normally. Since the ANOVA showed no differences, the results are consistent with those of Matarazzo et al. (1970). However, the comparison of the mean number of movements was not appropriate to detect the actual changes in movement that occurred while the students were lying. Some persons decrease their movement while lying, but other persons increase their movement.

In order to answer the question as to whether foot and leg movement changed while the student was lying, difference scores were computed for each person, and the absolute value of the difference score was used for the hypothesis tests. The values for the male students were compared to an expected change of zero, which would occur if there was no effect, using a t test. The $t$ value (13) of 4.048 led to rejection of the hypothesis of no difference (the mean change was 27.61 movements). A similar $t$ test, computed using the data for the females, produced a $t$ value (15) of 3.846 , which also led to rejection of the hypothesis (the mean change was 25.22 movements).

The change in movement data were inspected in an attempt to decide why some people increased their foot and leg movements when lying, while others decreased their movements. Table 2 contains a categorization of the amount of movement and the direction of the change for both male and female students. The amount

Table 2

The Numbers of Students Who Either Increased or Decreased Their Foot and Leg Movements While Lying

\begin{tabular}{|c|c|c|c|c|}
\hline \multirow{3}{*}{$\begin{array}{l}\text { Amount of } \\
\text { Movement }\end{array}$} & \multicolumn{2}{|c|}{ Males } & \multicolumn{2}{|c|}{ Females } \\
\hline & \multicolumn{2}{|c|}{$\begin{array}{c}\text { Direction of Change } \\
\text { of Movement }\end{array}$} & \multicolumn{2}{|c|}{$\begin{array}{c}\text { Direction of Change } \\
\text { of Movement }\end{array}$} \\
\hline & Increase & Decrease & Increase & Decrease \\
\hline Small & 3 & 2 & 1 & 4 \\
\hline Medium & 3 & 1 & 4 & 2 \\
\hline Large & 2 & 3 & 4 & 1 \\
\hline
\end{tabular}

Note-Students are categorized according to the total amount of change in movement. Small $=0.9$, medium $=9.5-21.5$, large $=35-84.5$. 
of change was categorized as small, medium, or large. The degree of movement represented by the words was chosen because the amounts separated the students into nearly equal groups for each category. Chi-squared values were computed for the data in Table 2 , and both fell into the acceptance region.

\section{DISCUSSION}

The suggestion of Ekman and Friesen (1969) that foot and leg movements, being more or less out of sight and mind, may betray the fact that a person is lying has been supported. When the changes in the amount of such movements were measured, the changes were large.

The use of videotape equipment to record the movements provided responses that could reliably be converted to numbers.

Even while many attempts at detecting whether people were lying, without connecting electronic equipment, have not been successful (e.g., Olechowski, 1967), the present procedure seems to be workable. Since counselors, interviewers, and therapists cannot usually connect their clients to detecting machines, but there is considerable interest in the veracity of their statements, the present method may be useful.

\section{REFERENCE NOTE}

1. McIntyre, L., \& Kintz. B. L. The effect of high and low topic suliency on interviewee speech and leg/foot movement rates. Unpublished manuscript, 1975.

\section{REFERENCES}

Burns, J. A., \& Kintz, B. L. Eye contact while lying during an interview. Bulletin of the Psychonomic Society, 1976, 7, 87-89.

Christie, R., \& Geis, F. L. (Eds.). Studies in Machiavellianism. New York: Academic Press, 1970.

Ekman, P., \& Friesen, W. V. Nonverbal leakage and clues to deception. Psychiatry, 1969, 32, 88-106.

Exline, R. V.. Thibaut, J., Hickey, C. B., \& Gumpert, P. Visual interaction in relation to Machiavellianism and an unethical act. In R. Christie \& F. L. Geis (Eds.), Studies in Machiavellianism. New York: Academic Press, 1970.

Hartshorne, H.. \& May, M. A. Studies in deceit. New York: Macmillan, 1928.

KINTZ, B. L. Lying on a test and in the laboratory. Bulletin of the Psychonomic Society, 1975, 6, 207-209.

Matarazzo. J. D., Wien, A. N., Jackson, R. H., \& Manaugh, T. S. Interviewee speech behavior under conditions of endogeneously-present and exogeneously-induced motivational states. Journal of Clinical Psychology, 1970, 26, 141-148.

Olechowski, R. Experimente über den Stimm, und Sprechausdrück beim Lügen. Zeitschrift für experimentelle und angewandte Psychologie, 1967, 14, 474-482.

(Received for publication August 8, 1977.) 